\title{
DEVELOPMENT OF A NEW WAVEGUIDE VALVE USING RF CHOKE FLANGE FOR PLS LINAC *
}

\author{
W. H. Hwang, H. S. Lee, S. H. Kim, Y. J. Park, and S. H. Nam \\ Pohang Accelerator Laboratory, POSTECH, Pohang 790-784, Korea
}

\begin{abstract}
Waveguide valves capable of handling a peak power of $80 \mathrm{MW}$ are required at the time of klystron replacement in PLS Linac. We designed a U-shaped waveguide valve and made two prototypes. However, they have some problems caused by rf power leak from the waveguide to the vacuum enclosure. This caused an increase of vacuum pressure and arcing in the vacuum chamber at the power level of $60 \mathrm{MW}$. A new choke flange has been designed with MAFIA code to solve problems caused by the rf power leak. A model choke flange has been fabricated and tested with a Network Analyzer and a $600 \mathrm{~W}$ solid state amplifier. It was observed that the rf leak at the choke flange can be reduced more than $20 \mathrm{~dB}$ in comparison with no choke case. High power rf test for the new waveguide valve with rf choke flange was performed in Sband resonant ring. The $\mathrm{rf}$ transmission through the valve was achieved up to $125 \mathrm{MW}$ peak power level at the pulse width of $4 \mu \mathrm{s}$ and the repetition rate of $30 \mathrm{~Hz}$.
\end{abstract}

\section{INTRODUCTION}

The PLS linac is a full energy injector to the storage ring for the third generation synchrotron source. The maximum klystron output power level is $80 \mathrm{MW}$ for $4 \mu \mathrm{s}$. When it occurs to replace a klystron due to klystron failure, the beam service should be stopped and about a week period is needed to recover the operating condition of linac. It is necessary to serve the beam during the replacement and to minimize the recovering time.

In order to solve this problem, the slac uses waveguide valves, and the Spring-8 linac, $1 \mathrm{GeV}$ linac, uses rf windows to separate the vacuum between the klystron waveguide and accelerating structures. The Viton-seal type waveguide valves were developed at SLAC, and they have been used quite a long period with good reliability for $\sim 65 \mathrm{MW}$ peak power with $3.5 \mu$ s pulse width. A new waveguide valve for $80 \mathrm{MW}$ at $4 \mu$ s has been designed by PLS with the collaboration work with IHEP, China. The prototype of the U-type waveguide valve (Figure 1) was fabricated and the high power test was conducted by using the resonant ring in the PLS. The vacuum pressure suddenly went up at the rf power of $60 \mathrm{MW}$ with $3.5 \mu \mathrm{s}$ pulse width at a repetition rate of $30 \mathrm{~Hz}$ [1].

We found a few problems in the U-type valve. The first is the increase of the vacuum pressure in the chamber caused by the rf leak. The second is arcing problems occurred at the inside of the chamber and the waveguide rf contact point. The last is the different vacuum pressure between the inside and the outside of the waveguide in the chamber. All problems is caused by the rf leak which is from the incomplete rf contact.

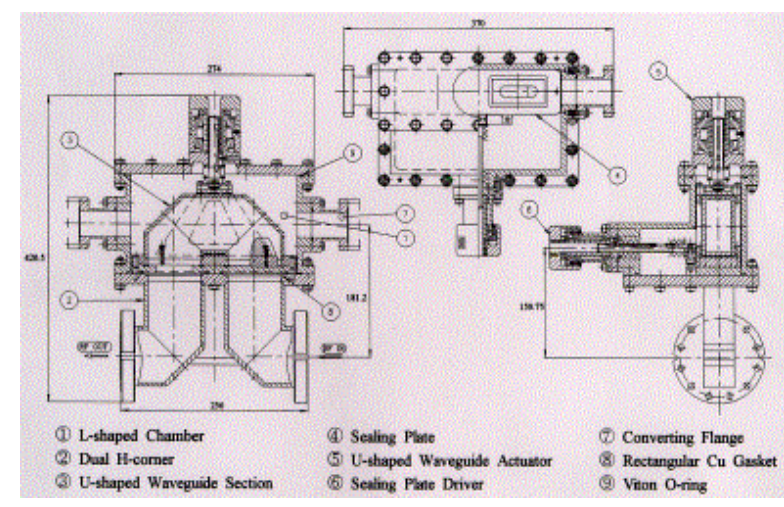

Figure 1: U-type waveguide valve

\section{DESIGN OF CHOKE FLANGE}

We studied how to minimize the leak power into the chamber and keep the same vacuum pressure of the chamber as the pressure of the waveguide. The slac waveguide valve shows that the maximum tolerable rf leak power was set at 2 watts peak in the O-ring chamber [2]. To get this value, the ratio of the transmitted power into the waveguide valve to the leak power into the chamber should be greater than $80 \mathrm{~dB}$ for operation of 100 MW. A flange with a choke to seal the rf was designed with the MAFIA simulation code to achieve the $80 \mathrm{~dB}$ isolation and to solve the problem of the different vacuum pressure between the chamber and the waveguide.

There are four parameters to be determined in the design of a choke flange: position, depth, width of the choke and the gap between flanges. We choose the gap distance of $1 \mathrm{~mm}$ and choke width of $10 \mathrm{~mm}$, respectively. The gap distance affect on the rf reflection ratio (vswr) in the waveguide. In case of $d=1 \mathrm{~mm}$, the calculated vswr is less than 1.1, even if all leak power is reflected back [3]. But the choke width was decided arbitrarily. And the other parameters were decided by computer simulation. The simulation results are shown in Figure 2 and Figure 3. We can determine the choke dimension with these results. The choke position for the operating frequency can be determined from Figure 2 and the choke depth from the relationship of these two parameters as shown in Figure 3.

*Supported by the Ministry of Science and Technology, Korea 


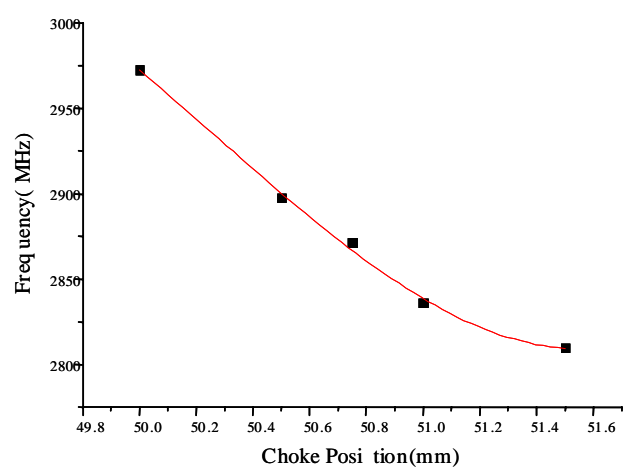

Figure 2: Relation between choke position and resonant frequency

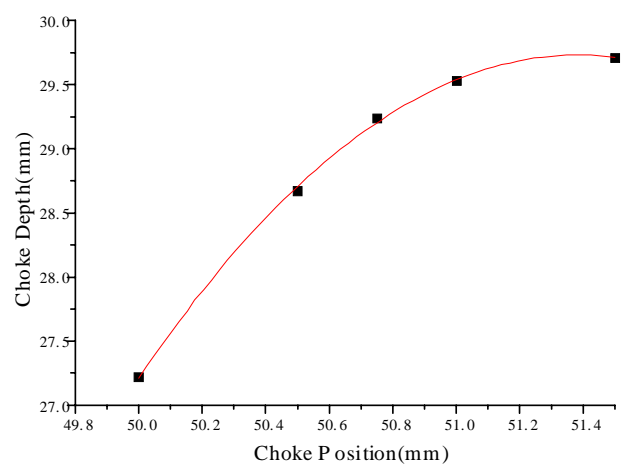

Fig.3. Relation between choke position and choke depth $(\mathrm{d}=1 \mathrm{~mm})$

From the computer simulation results we obtained the choke dimensions of choke depth $29.31 \mathrm{~mm}$, choke position $50.84 \mathrm{~mm}$, choke width $10 \mathrm{~mm}$, and gap distance $1 \mathrm{~mm}$ for the operating frequency of $2856 \mathrm{MHz}$. Also, in the simulation by HFSS code for choke flange with the dimensions, the ratio of a transmitted power into the waveguide valve to a leak power into the chamber is $75 \mathrm{~dB}$ and return loss is $47 \mathrm{~dB}$. In a case of flat flange, the ratio and return loss is $48 \mathrm{~dB}$ and $33 \mathrm{~dB}$ respectively. By using the choke flange, the leak power into the chamber is decreased about $27 \mathrm{~dB}$ more than a flat flange.

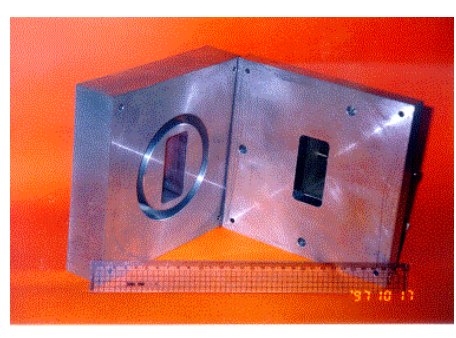

(a)

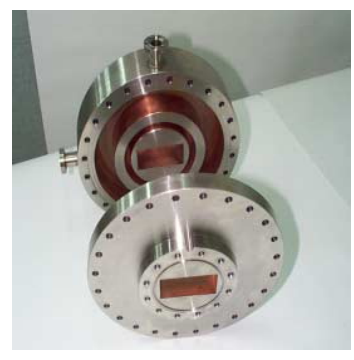

(b)
Figure 4: (a) A model choke flange for rf cold test

(b) A proto type choke flange for if hot test

A model choke flange was fabricated (Figure 4a) and tested. We measured the rf leak power using a network analyser (HP8510C). The leak power at the flange gap was decreased about $28 \mathrm{~dB}$ by using the choke flange. The measured values are nearly same as the calculated values by HFSS code.

Based on the positive cold test results, we fabricated a prototype choke flange for high power test. The flange was made of stainless steel and its inner wall was plated with copper for a better rf transmission (Figure 4b).

We performed a high power rf test to investigate the high power rf characteristics of the choke flange. The test was carried out using a S-band resonant ring facility (Figure 5), which consisted of modulator and klystron system, waveguide system, vacuum and cooling system, several $\mathrm{rf}$ analyzer. The temperature of the waveguide network in the resonant ring was controlled within $0.1^{\circ} \mathrm{C}$ at $32^{\circ} \mathrm{C}$ by using a precision temperature controller. In the test, rf transmission of the choke flange showed no breakdown up to $150 \mathrm{MW}$ peak power level of $3 \mu$ s pulse width at a repetition rate of $30 \mathrm{~Hz}$. Figure 6 shows a waveform of the klystron output and choke flange input with $3 \mu$ s pulse width.

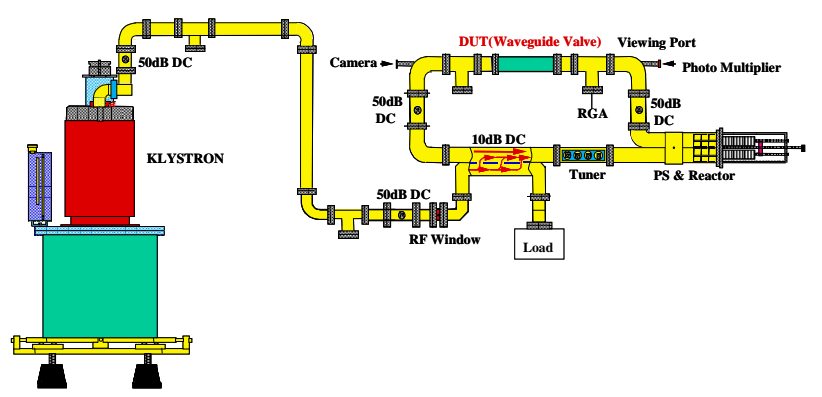

Figure 5: Layout of high power test for waveguide valve

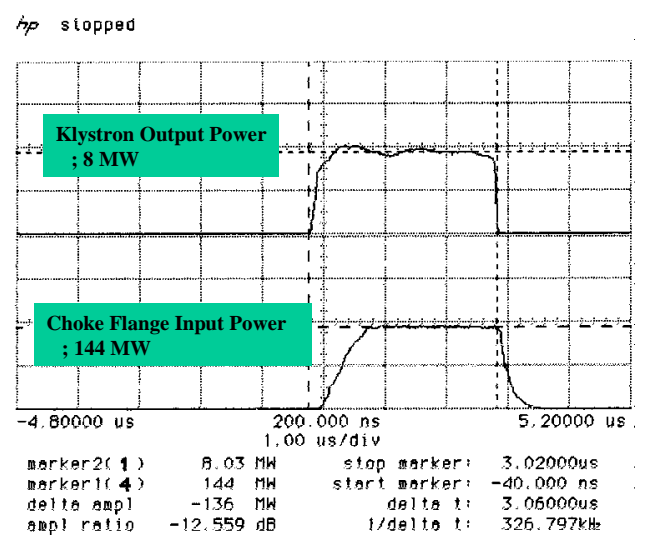

Fig. 6. Waveform of klystron output and Choke flange input

\section{WAVEGUIDE VALVE}

Considering the rf seal, simple structure, easy push-pull type handle, mechanical alignment and pin holes to easy 
gas flow, a new waveguide valve was designed and fabricated as shown in Figure 7.
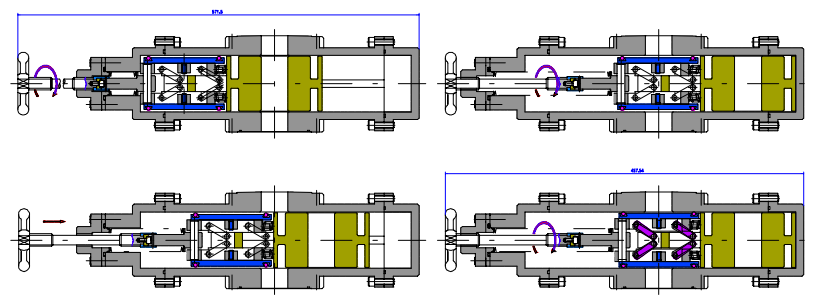

Figure 7: Waveguide valve using rf choke flange

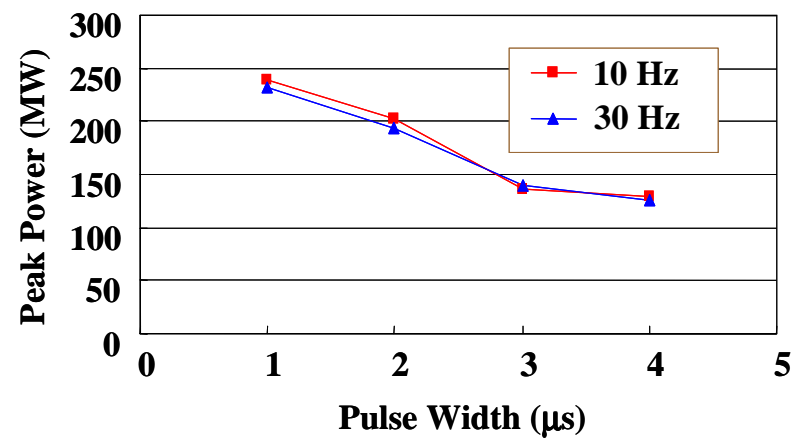

Figure 8: Maximum allowable power level of waveguide valve

In cold test by using network analyser, vswr was 1.03 and insertion loss was $0.04 \mathrm{~dB}$ at $2856 \mathrm{MHz}$. The high power test for the waveguide valve has been performed in S-band travelling resonant ring. At the starting point, the rf power of $1 \mathrm{MW}$ with $0.9 \mu$ s width at a repetition rate of $10 \mathrm{~Hz}$ was applied to the valve. At that condition, the vacuum pressure in resonant ring was $1.3 \times 10^{-7}$ Torr and the return loss of the valve was $20 \mathrm{~dB}$. We increased the power level at the same pulse width and repetition rate to investigate the breakdown phenomena in the ring with photo multiplier. At the power of $239 \mathrm{MW}$, the vacuum pressure was $4.7 \times 10^{-8}$ Torr, and the return loss was $21 \mathrm{~dB}$. RF performance tests were conducted according to test procedure. The power level, rf pulse width and repetition rate was carefully increased. At the power of $125 \mathrm{MW}$ with $4 \mu$ s and $30 \mathrm{~Hz}$, the vacuum was $3.7 \times 10^{-8}$ Torr and the return loss was $21 \mathrm{~dB}$. But, the breakdown occurred above power of $125 \mathrm{MW}$. Figure 8 shows the maximum endurable power level of the waveguide valve.

\section{SUMMARY}

Waveguide valves of $80 \mathrm{MW}$ of handling power are needed for the replacement of klystrons in PLS Linac. We have designed a new type of waveguide valve using a $\mathrm{rf}$ choke flange to improve rf leak troubles from the uneven contact between flanges. The rf leak at the choke flange of waveguide valve was reduced more than $20 \mathrm{~dB}$ in comparison with no choke case. In high power test, no rf breakdown occurred below power level of $125 \mathrm{MW}$ of peak power with $4 \mu$ s width. The test results indicate that the new valve can function well up to the maximum power level of PLS linac klystron; peak power of with pulse width of $4 \mu$ s.

\section{REFERENCES}

[1] J. S. Bak, W. Namkung, Journal of the Korean Vacuum Society, vol. 4, No.2, 124(1995)

[2] N. R. Dean, W. R. Fowkes, M. W. Hoyt, H. D. Schwarz and E. F. Tillman, IEEE Trans. On Nucl. Sci. NS-33, 1611(1987)

[3] New design of waveguide valve with choke cavity in Proc. 1944 KAPRA \& KPS/DPP Joint Workshop, June 27-28 (1996) 